\title{
Study on Surface Integrity of AISI 1045 Carbon Steel when machined by Carbide Cutting Tool under wet conditions
}

\author{
N. Fauzi Tamin ${ }^{1, *}$, U.A.A. Azlan ${ }^{1}$, M. Hadzley ${ }^{2}$, N.S. Remle ${ }^{1}$, M. Hairizal Osman ${ }^{1}$, \\ M. Rahimi Yusoff ${ }^{1}$, and N.Z. Noriman ${ }^{3}$ \\ ${ }^{1}$ Faculty of Engineering Technology, Universiti Teknikal Malaysia Melaka (UTeM), Hang Tuah Jaya, \\ 76100 Durian Tunggal, Melaka, Malaysia \\ ${ }^{2}$ Faculty of Manufacturing Engineering, Universiti Teknikal Malaysia Melaka (UTeM), Hang Tuah \\ Jaya, 76100 Durian Tunggal, Melaka, Malaysia \\ ${ }^{3}$ Faculty of Engineering Technology, Universiti Malaysia Perlis (UniMAP), Aras 1, Blok S2, Kampus \\ UniCITI Alam Sungai Chuchuh, Padang Besar, 02100 Perlis, Malaysia
}

\begin{abstract}
This paper presents the evaluation of surface roughness and roughness profiles when machining carbon steel under wet conditions with low and high cutting speeds. The workpiece materials and cutting tools selected in this research were AISI 1045 carbon steel and canela carbide inserts graded PM25, respectively. The cutting tools undergo machining tests by $\mathrm{CNC}$ turning operations and their performances were evaluated by their surface roughness value and observation of the surface roughness profile. The machining tests were held at varied cutting speeds of 35 to 53 $\mathrm{m} / \mathrm{min}$, feed rate of 0.15 to $0.50 \mathrm{~mm} / \mathrm{rev}$ and a constant depth of cut of $1 \mathrm{~mm}$. From the analysis, it was found that surface roughness increased as the feed rate increased. Varian of surface roughness was suspected due to interaction between cutting speeds and feed rates as well as nose radius conditions; whether from tool wear or the formation of a built-up edge. This study helps us understand the effect of cutting speed and feed rate on surface integrity, when machining AISI 1045 carbon steel using carbide cutting tools, under wet cutting conditions.
\end{abstract}

\section{Introduction}

Carbon steel is an engineering material that is generally contained with steel and carbon. The properties of carbon steel depend on the carbon contained within their composition, ranging from low $(0.05-0.2 \%)$, medium $(0.30-0.60 \%)$ and high $(0.7-2.5 \%)$ [1]. Carbon steel possesses several important characteristics, such as being difficult to weld, bend, machine and cut; depending on its properties. When heat treatment is applied, the properties of carbon steel can be altered whereby it can change the formability, machinability and weldability of the products [2]. Carbon steel is considered to be an important alloy used in industry, since it accounts for over $90 \%$ of all construction materials, automotive, petroleum and chemical industries [3]. One of the dominant processes used to produce the

\footnotetext{
*Corresponding author: norfauzi@utem.edu.my
} 
parts made from carbon steel is machining. Carbon steel is still being machined, as this material possesses good characteristics during material removal and is considered soft and rigid when engaged with a cutting tool. During workpiece-cutting tool engagement, contact at the tool-chip and tool-workpiece interfaces generates heat; and therefore, wet cutting is generally applied to enhance machining performance. The use of wet cutting enables the machining process to obtain maximum metal removal and accurate dimensions; thus achieving an adequate surface quality.

Despite the advantages of machining carbon steel in wet conditions, there are some difficulties machining this material; especially when the cutting parameters are not applied correctly. Existing industry cutting tools usually facilitate guidance parameters for general machining practices. The use of specific cutting tools for specific materials; especially in wet conditions, still need to be explored - as it can enhance the machinist's knowledge to observe the behaviour of material during machining and apply parameters correctly. This paper investigates the surface roughness when machining carbon steel AISI 1045 in wet conditions at different cutting speeds, feed rates and a constant depth of cut. In this study, a 3 axis CNC lathe was used for machining trials. The surface integrity of carbon steel was evaluated using a surface roughness tester. The data obtained from this study will be useful as a reference for machinists planning their machining, in order to produce maximum surface quality, reduce processing steps and minimize operational costs.

\section{Experimental procedures}

This work aims to identify the surface roughness and roughness profile of carbon steel at several cutting speeds and feed rates in wet conditions. The experiments were carried out using a CNC Machine (DMG-CTX 310). The medium, carbon steel of AISI 1045, was selected as the workpiece material. The types of carbide tools used in this work were made by SANDVICK-Coromat (as shown in Fig. 1). The tool holder used was a DCLNL 2020K (as shown in Fig. 2). Pre-tech mineral oil was used as a coolant. A surface roughness tester (Mitutoyo SJ-410) was used to determine the surface roughness value and roughness profiles. Table 1 show the cutting parameters used in this study.

Table 1. Cutting conditions for machining tests (Depth of cut: constant $1 \mathrm{~mm}$ )

\begin{tabular}{|c|c|c|}
\hline No. & Cutting speed (m/min) & Feed rate (mm/rev) \\
\hline 1 & 35 & 0.15 \\
\hline 2 & 41 & 0.15 \\
\hline 3 & 47 & 0.15 \\
\hline 4 & 53 & 0.15 \\
\hline 5 & 35 & 0.50 \\
\hline 6 & 41 & 0.50 \\
\hline 7 & 47 & 0.50 \\
\hline 8 & 53 & 0.50 \\
\hline
\end{tabular}




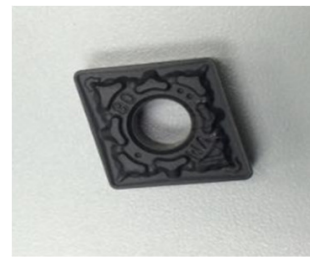

Fig. 1. SANDVICK Coromat cutting tool

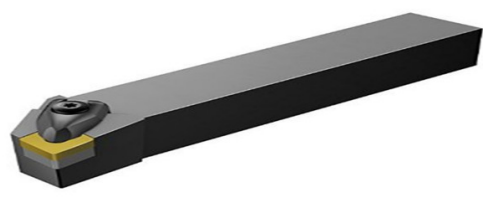

Fig. 2. Tool Holder DCLNL 2020K

\section{Results and discussion}

Fig. 3 shows a plot of surface roughness ( $\mathrm{Ra}$ ) based on the determined cutting speed and feed rates. It shows the line graph of the surface roughness, Ra versus cutting speed and surface roughness data, $\mathrm{Ra}$ in wet conditions at different cutting speeds with a constant feed rate of $0.15 \mathrm{~mm} / \mathrm{rev}$. At a cutting speed of $35 \mathrm{~m} / \mathrm{min}$, the value of surface roughness was $3.085 \mu \mathrm{m}$, increasing to $3.948 \mu \mathrm{m}$ at $47 \mathrm{~m} / \mathrm{min}$; but decreasing to $3.283 \mu \mathrm{m}$ at $53 \mathrm{~m} / \mathrm{min}$. Fig. 4 shows the $0.50 \mathrm{~mm} / \mathrm{rev}$ with different cutting speeds. The line graph shows that surface roughness, Ra decreased slightly for the following cutting speeds; $35 \mathrm{~m} / \mathrm{min}, 41$ $\mathrm{m} / \mathrm{min}, 47 \mathrm{~m} / \mathrm{min}$ and decreased even more at a cutting speed of $53 \mathrm{~m} / \mathrm{min}$. Surface roughness values following these cutting speeds were $16.327 \mu \mathrm{m}, 15.774 \mu \mathrm{m}, 15.022 \mu \mathrm{m}$ and $14.923 \mu \mathrm{m}$, respectively. Thus, surface roughness decreased with an increased cutting speed. It should be noted that the Ra recorded at lower feed rate of $0.15 \mathrm{~mm} / \mathrm{rev}(3.085-$ $3.948 \mu \mathrm{m})$ are so much lower than higher feed rate of $0.5 \mathrm{~mm} / \mathrm{rev}(14.923-16.327 \mu \mathrm{m})$. This shows that the feed rate is the most influencing parameter affecting on surface roughness. The results consistent with the review from [4] where the surface finish obtained is good when the lower feed rate is applied as compared to higher feed rate. Low surface roughness enables to produce accurate dimension and increase the fatigue life of the machined components [5].

Fig. 5 shows the surface roughness profile at feed rate $0.15 \mathrm{~mm} / \mathrm{rev}$ with different cutting speeds. It shows that at the lower cutting speed of $35 \mathrm{~m} / \mathrm{min}$, the surface roughness profile showed uniform feed marks according to the nose radius engagement of the cutting tool at the upper machined surface. As the cutting speed increased to $41 \mathrm{~m} / \mathrm{min}$ and 47 $\mathrm{m} / \mathrm{min}$, the distance between feed marks became closer; hence, resulting in a higher surface roughness value (as shown in Fig. 3). However, as the cutting speed increased to $53 \mathrm{~m} / \mathrm{min}$, the surface roughness profile exhibited an almost flat appearance. It is suspected that at the highest cutting speed of $53 \mathrm{~m} / \mathrm{min}$, the cutting tool was already worn, which had altered the nose radius of the cutting tool, thus resulting in an alteration of feed marks to an almost flat appearance on the roughness profile. Another possible mechanism was the presence of a built-up edge that altered the nose radius of the cutting tool. A built-up edge is the formation of molten metal that adheres to the nose radius due to high temperatures generated at the tool-chip interface [6]. The existence of a built-up edge may indicate that the coolant did not penetrate the cutting zone due to the higher cutting speed or feed rate that splashing away the coolant.

Fig. 6 shows the surface roughness profile for a feed rate of $0.50 \mathrm{~mm} / \mathrm{rev}$. It shows that every different cutting speed had different surface roughness profiles. The surface profile of the presented figures shows a larger distance between feed marks (compared to Figure 5) due to the larger distance of feed rate. The surface profile exhibited fine feed marks at a lower cutting speed of $35 \mathrm{~m} / \mathrm{min}$. As the cutting speed increased to $41 \mathrm{~m} / \mathrm{min}$ and 47 $\mathrm{m} / \mathrm{min}$, the feed marks exhibited finer distance of peak and valley, resulting in a lower surface roughness (as shown in Fig. 4). It should be noted however, as the cutting speed 
increased to $53 \mathrm{~m} / \mathrm{min}$, the surface roughness profile demonstrated smaller gaps between the feed marks, may be due to several aspects that need to further investigated.

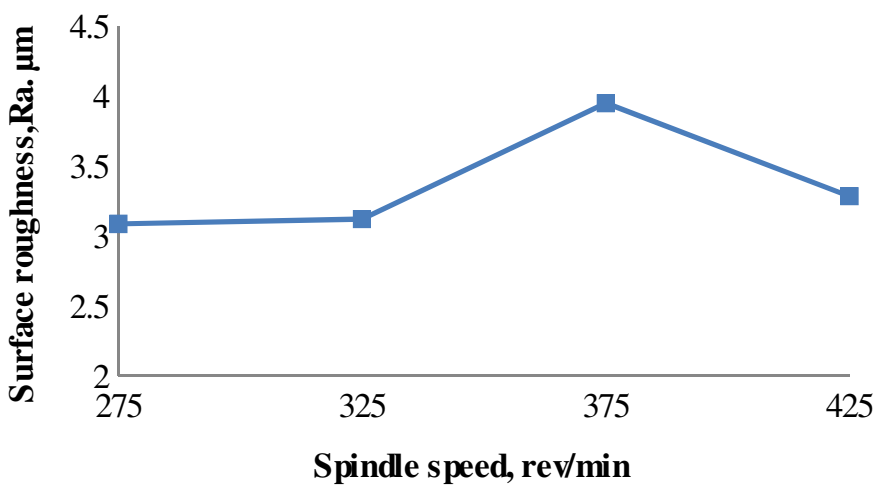

Fig. 3. Surface roughness vs. cutting speed using feed rate $0.15 \mathrm{~mm} / \mathrm{rev}$

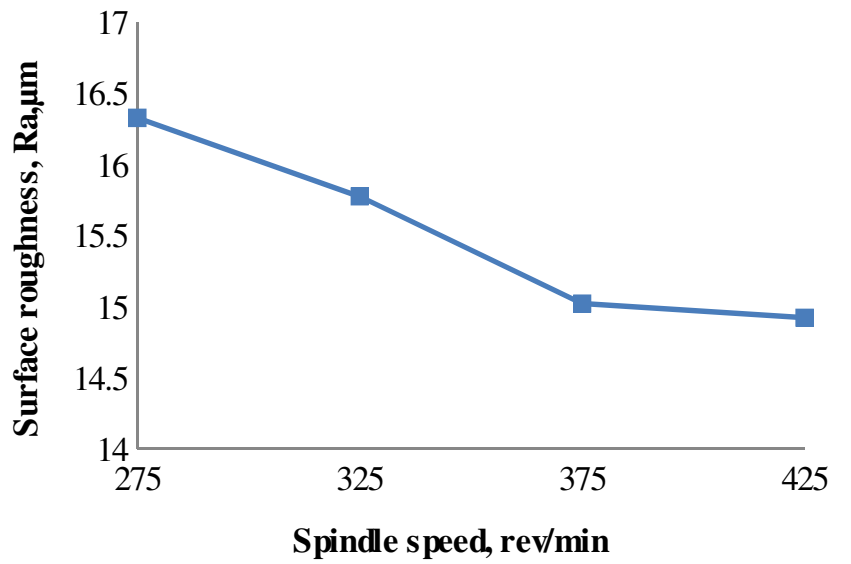

Fig. 4. Surface roughness vs. cutting speed using feed rate $0.50 \mathrm{~mm} / \mathrm{rev}$

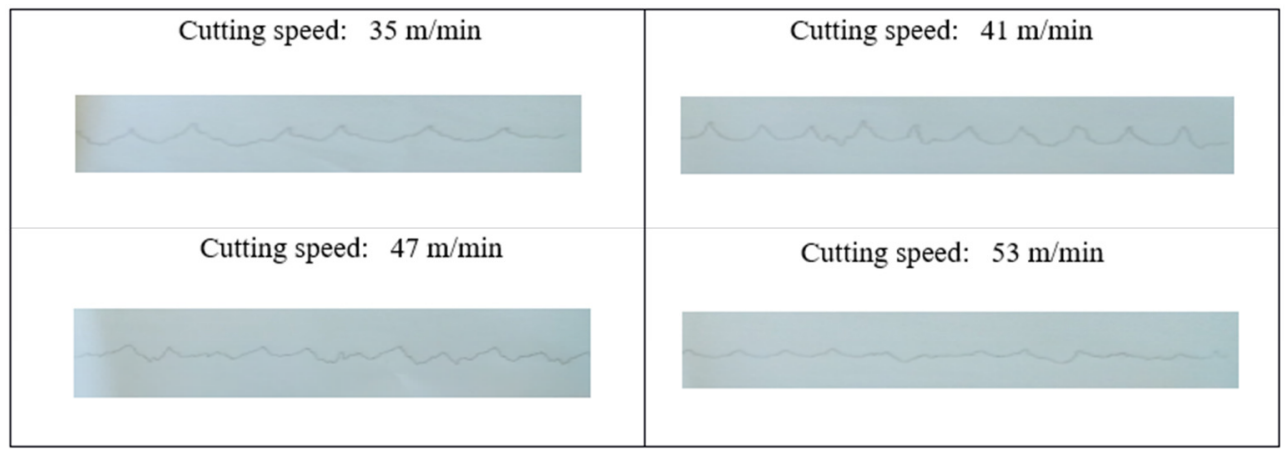

Fig. 5. Surface roughness profile at feed rate $0.15 \mathrm{~mm} / \mathrm{rev}$ 


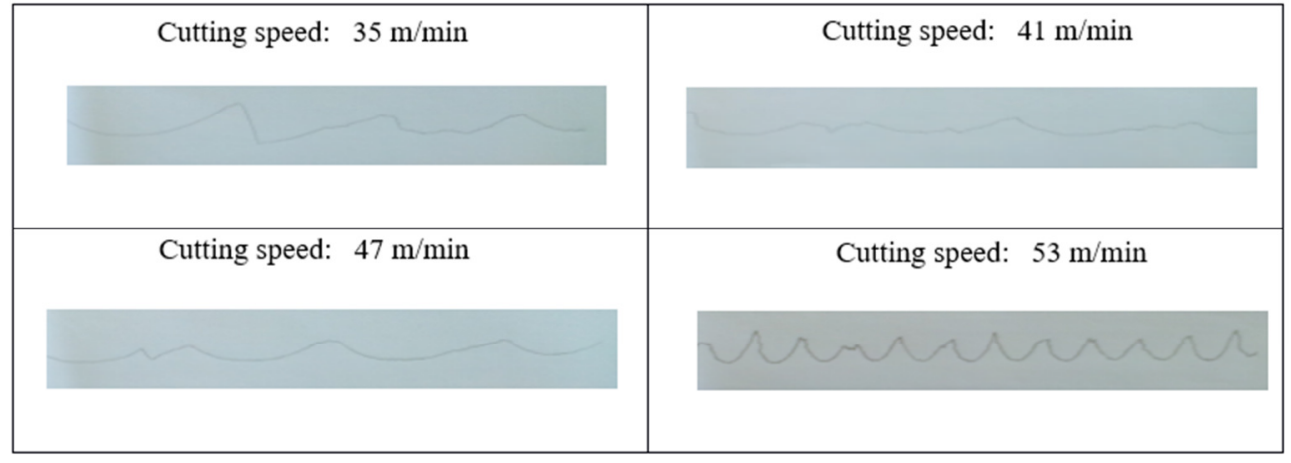

Fig. 6. Surface roughness profile at feed rate $0.50 \mathrm{~mm} / \mathrm{rev}$

\section{Conclusion}

This paper presents the experimental of surface roughness of Carbon Steel AISI 1045 using wet cutting CNC turning. From the machining data collected, and based on the experimental findings, the following conclusions were made:

i. The surface roughness increased when the cutting speed was increased at a feed rate of $0.15 \mathrm{~mm} / \mathrm{rev}$.

ii. The surface roughness decreased when the cutting speed was increased at a constant feed rate with a value of $0.50 \mathrm{~mm} / \mathrm{rev}$.

iii. The use of lower feed rates is suitable for wet cutting.

iv. This study helps us to understand the effects of cutting speed and feed rate on the surface roughness of Carbon Steel AISI 1045 under wet cutting conditions.

The author would like to thank Universiti Teknikal Malaysia Melaka and Ministry of Higher Education under the Fundamental Research Grants Scheme (FRGS) number: FRGS/1/2014/TK04/FTK/02/F00207 for funding this project.

\section{References}

1. J.K. Odusote, T.K. Ajiboye, A.B. Rabiu, J. Miner, Mater. Charact. Eng., 11 (2012)

2. D. Gandy, Carbon Steel Handbook. Paolo Alto: Electric Power Research Institute. (2007)

3. S.O. Seidu, B.J. Kutelu, J. Miner, Mater. Charact. Eng., 1 (2013)

4. S.B. Chikalthankar, R.B. Kakade, V.M. Nandedkar, Int. J. Eng. Res. Technol., 3, 10 (2014)

5. A.B. Mohd Hadzley, M.R. Nurul Fatin, R.A. Raja Izamshah, N.I.S. Hussein, A. Siti Sarah, Sivarao, Appl. Mech. Mater., 465-466 (2014)

6. A.B. Mohd Hadzley, A. Siti Sarah, R.A. Raja Izamshah, A.A. Mohd, M.S. Kasim, M.A. Sulaiman, M.R. Nurul Fatin, Appl. Mech. Mater., 465-466 (2014) 